\title{
A NOTE ON THE GENERALIZED (POSITIVE) CAUCHY DISTRIBUTION
}

\author{
B. Raja Rao and M. L. Garg
}

In this note, a certain generalization of the Cauchy distribution is obtained, using the result of Malik [2]. density

1. Introduction. The generalized gamma distribution having the

$$
f(x, a, d, p)=\frac{p}{\Gamma(d / p) a^{d}} x^{d-1} e^{-(x / a)^{p}}, x>0 ; a, d, p>0
$$

is introduced by Stacy [1], who studied some of its properties. As remarked by Stacy [1], the familiar gamma, chi, chi-squared, exponential and Weibull distributions are special cases of (1), as are the distributions of certain functions of a normal variable - viz., its positive even powers, its modulus, and all positive powers of its modulus.

Malik [2] obtained the distribution of the ratio $\mathrm{W}=\mathrm{X} / \mathrm{Y}$ where $\mathrm{X}$ and $Y$ are independent random variables distributed according to (1) with parameters $\left(a_{1}, d_{1}, p\right)$ and $\left(a_{2}, d_{2}, p\right)$. The density of $W$ is (see $\left.[2, E q . .2 .6]\right) *$

(2) $g(w)=$

$$
\frac{p\left(\frac{a_{1}}{a_{2}}\right)^{d}}{B\left(\frac{1}{p}, \frac{2}{p}\right)}
$$$$
\frac{w^{-d_{2}-1}}{\left[1+\left(\frac{a_{2}}{a_{1}}\right)^{-p} w^{-p}\right]^{\frac{d_{1}+d_{2}}{p}}}, w>0 \text {. }
$$

It is the purpose of this note to examine Stacy's remark as applied to the distribution of $\mathrm{W}$ and to obtain a generalized Cauchy distribution.

Incidentally Malik's result (2) may be called a generalization of the beta distribution of the second kind, to which it will reduce if we specialize to $\mathrm{p}=1$ and $\mathrm{a}_{1}=\mathrm{a}_{2}$.

2. The generalized Cauchy and other allied distributions. It is well known that the ratio of two independent normal random variables has a Cauchy distribution. Further, the ratio of the moduli of two independent normal random variables $U=|X| /|Y|$ has the (positive) Cauchy distribution with the density

*Malik's Eq. 2.6 is slightly incorrect. The factor $\log \left(\frac{a_{1}}{a_{2}}\right)$ in the exponent of the denominator is to be multiplied by $\mathrm{p}$. 


$$
p(u)=\frac{2}{\pi} \frac{1}{\left(1+u^{2}\right)}, \quad u \geq 0 .
$$

To pursue Stacy's remark, let $\mathrm{X}$ be distributed according to $\mathrm{N}(0,1)$. Then the distribution of $Z=|X|^{\ell}$ for $\ell>0$ is

$$
g(z)=\frac{2}{\ell \sqrt{(2 \pi)}} z^{\frac{1}{\ell}-1}\left\{\exp -\frac{1}{2} z^{2 / l}\right\} z \geq 0
$$

which is in Stacy's form $f\left(z, 2^{\frac{1}{2} l}, \frac{1}{l}, \frac{2}{\ell}\right)$.

Now let $\mathrm{x}_{1}$ and $\mathrm{x}_{2}$ be independent standard normal variables and write

$$
\mathrm{Y}=\left|\mathrm{x}_{1}\right|^{\ell} \text { and } \mathrm{Z}=\left|\mathrm{X}_{2}\right|^{\ell}, \quad \ell>0
$$

Then $\mathrm{Y}$ and $\mathrm{Z}$ are independently and identically distributed according to Stacy's form $\mathrm{f}\left(\cdot, 2^{\frac{1}{2} \ell}, \frac{1}{\ell}, \frac{2}{\ell}\right)$. If we now define

$$
\mathrm{W}=\frac{\mathrm{Y}}{\mathrm{Z}}=\left\{\frac{\left|\mathrm{x}_{1}\right|}{\left|\mathrm{x}_{2}\right|}\right\}^{\ell}
$$

the density of $\mathrm{W}$ is obtained from Malik's equation 2.6 (our equation (2)) by choosing in particular

$$
a_{1}=a_{2}=2^{\frac{1}{2} l}, \quad d_{1}=d_{2}=\frac{1}{l}, \quad p=\frac{2}{l} .
$$

Thus the density of $\mathrm{W}$ is

$$
g(w)=\frac{2}{l B\left(\frac{1}{2}, \frac{1}{2}\right)} \cdot \frac{w^{\frac{1}{l}-1}}{\left[1+w^{2 / \ell}\right]}, \quad w>0 .
$$

866 
For $\ell=1$, this reduces to equation (3). Thus (5) may be called the generalized (positive) Cauchy distribution.

Next, a generalization of the Beta distribution of the first kind, analogous to (2), may be obtained as follows. Let $X$ and $Y$ be independently distributed according to (1) with parameters $\left(a_{1}, d_{1}, p\right)$ and $\left(a_{2}, d_{2}, p\right)$.

If we define

$$
V=\frac{X}{X+Y}=\frac{1}{1+\frac{Y}{X}}=\frac{1}{1+W^{\prime}} \text { with } W^{\prime}=\frac{Y}{X} \text {, }
$$

the distribution of $W^{\prime}$ is obtained from (2) by interchanging the suffixes 1 and 2 as

(2') $\quad g\left(w^{\prime}\right)$

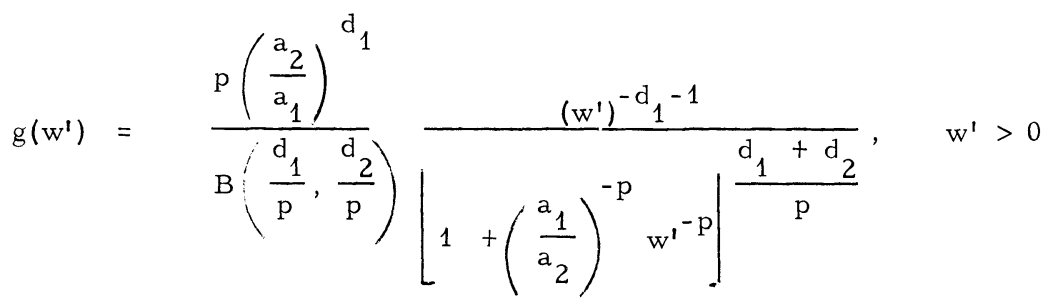

so that the density of $\mathrm{V}$ is

(6) $\mathrm{p}(\mathrm{v})=$

$$
\frac{p\left(\frac{a_{2}}{a_{1}}\right)^{d_{1}}}{B\left(\frac{d_{1}}{p}, \frac{d_{2}}{p}\right)}\left[1+\left(\frac{a_{2}}{a_{1}}\right)^{p}\left(\frac{v}{1-v}\right)^{p}\right]^{\frac{d_{1}+d_{2}}{p}}, 0 \leq v \leq 1 .
$$

For $a_{1}=a_{2}$ and $p=1$, (6) reduces to the beta distribution of the first kind.

Acknowledgement. The authors wish to thank Professor A. Ciocco for his constant stimulating encouragement. 


\section{REFERENCES}

1. E.W. Stacy, A generalization of the gamma distribution. Ann. Math. Statist. 33 (1962) 1187-1192.

2. H.J. Malik, Exact distribution of the quotient of independent generalized gamma variables. Canad. Math. Bull. 10 (1967) $463-465$.

Departments of Biostatistics and Mathematics

University of Pittsburgh

Pittsburgh

Pennsylvania 15213

Department of Biometrics

Temple University School of Medicine

Philadelphia, Pa. 19140 